\title{
Ein systematischer Backstepping-Zugang zur Regelung gekoppelter ODE-PDE-ODE-Systeme
}

\author{
A systematic backstepping approach for the control of coupled ODE-PDE-ODE systems
}

https://doi.org/10.1515/auto-2020-0030

Empfangen 29. Februar 2020; angenommen 9. Juni 2020

Zusammenfassung: Der systematische Zugang zum Backstepping-Entwurf von Zustandsrückführungen für gekoppelte ODE-PDE-ODE-Systeme, die sich zumeist für verteilt-parametrische Prozesse unter Berücksichtigung der Dynamik von Aktoren und Sensoren ergeben, erlaubt nicht nur die sukzessive und vereinfachte Herleitung von bereits bekannten Backstepping-Reglern sondern ermöglicht auch deren Entwurf für bisher nicht betrachtete Systemklassen. Der Zugang nutzt dabei die diesen Systemen inhärente strenge Rückkopplungsform aus. Wie beim klassischen Integrator-Backstepping wird das ODEPDE-ODE-System schrittweise durch virtuelle Zustandsrückführungen stabilisiert und der Zustand in Fehlerkoordinaten überführt. Der vorgeschlagene, modulare Entwurf ist dabei im Wesentlichen unabhängig davon, ob der verteilt-parametrische Teil des Systems parabolisch oder hyperbolisch ist.

Schlagwörter: verteilt-parametrische Systeme, parabolische Systeme, hyperbolische Systeme, Backstepping, rekursiver Reglerentwurf, Zustandsrückführung

\footnotetext{
Abstract: A systematic backstepping design of state feedback controllers is addressed for coupled ODE-PDE-ODE systems, that mostly arise for distributed-parameter systems where actuator and sensor dynamics are taken into account. It allows the successive and simplified derivation of known backstepping controllers and can easily be transferred to a broader spectrum of systems, that had not yet been considered. For that, the approach makes use of the strict feedback form that is inherent in these systems. Using integrator backstepping techniques, the ODE-PDEODE system is stabilized step-by-step by virtual state feedbacks and mapped into error coordinates. The proposed modular design is essentially independent of whether the

*Korrespondenzautor: Nicole Gehring, Johannes Kepler Universität, Institut für Regelungstechnik und Prozessautomatisierung, Altenberger Straße 69, 4040 Linz, Österreich, E-Mail: nicole.gehring@jku.at
}

distributed-parameter part of the system is parabolic or hyperbolic.

Keywords: distributed-parameter systems, parabolic systems, hyperbolic systems, backstepping, recursive feedback design, state feedback

\section{Einleitung}

Der im Folgenden vorgestellte systematische Backstepping-Entwurf von stabilisierenden Zustandsrückführungen für sogenannte ODE-PDE-ODE-Systeme bietet einen einheitlichen Rahmen sowohl für hyperbolische als auch für parabolische partielle Differentialgleichungen (PDEs), die wechselseitig mit gewöhnlichen Differentialgleichungen (ODEs) gekoppelt sind. Die parabolischen PDEs beschreiben dabei typischerweise Diffusionsphänomene, wie sie beispielsweise bei Wärmeleitungsproblemen oder chemischen bzw. biochemischen Reaktionsprozessen auftreten. Hingegen kommen hyperbolische PDEs meist dann zur Beschreibung eines Prozesses zum Einsatz, wenn Wellen und deren Ausbreitung mit endlicher Geschwindigkeit dominieren. Dazu zählen unter anderem elektrische, pneumatische oder anderweitig geartete Übertragungsleitungen und schwingende Saiten.

Unabhängig vom Charakter der PDE werden solche Systeme betrachtet, bei denen dynamische Randbedingungen auftreten. Diese führen zu einer beidseitigen Kopplung einer PDE oder eines PDE-Systems mit ODEs an den entsprechenden Rändern der PDE(s); es entstehen ODE-PDE-ODE-Systeme. Die ODEs beschreiben dabei unter anderem eine Sensor- oder Aktordynamik, können aber auch bei beliebigen anderen Kopplungen zwischen Prozessen mit verteilten und konzentrierten Parametern zum Vorschein kommen. Als Beispiel seien die Wechselwirkung zwischen (konzentrierten) Energieerzeugern und Energieverbrauchern über zumeist durch hyperbolische PDEs charakterisierte Übertragungsleitungen genannt oder auch einfach eine Last an einem schweren Seil. 
Die zentrale Herausforderung beim BacksteppingEntwurf von Reglern für PDE-Systeme besteht in der Wahl eines geeigneten Ansatzes für die BacksteppingTransformation sowie eines Zielsystems, in das transformiert werden soll und das aufgrund seiner einfachen Struktur leicht durch eine entsprechende Zustandsrückführung stabilisiert werden kann. Beides wird für ODEPDE-ODE-Systeme wesentlich erschwert. Obwohl diese Systemklasse in den letzten Jahren stark im Fokus von Wissenschaftlern auf dem Gebiet des Backstepping-Entwurfs stand, decken publizierte Ergebnisse oftmals nur Sonderfälle ab. So beschränkt sich [2] auf die Kopplung einer (skalaren) Transportgleichung mit ODEs erster Ordnung und [5] unter sehr restriktiver Annahmen auf zwei hyperbolische PDEs, die mit ODEs beliebiger Ordnung in Wechselwirkung stehen. Gleiches gilt für parabolische Systeme, für die man sich für den Reglerentwurf zumeist auf eine einfache Wärmeleitungsgleichung beschränkt (z. B. [20] oder der PDE-ODE-Fall in [17]). Im Gegensatz zu diesen Einschrittentwürfen wird in [7] und [6] die Verwendung mehrerer Transformationsschritte vorgeschlagen, also ein Mehrschrittverfahren. Den einzelnen Entwurfsschritten ist dabei jeweils eine Aufgabe in Bezug auf die Stabilisierung und Entkopplung von Teilsystemen des ODEPDE-ODE-Systems zugeordnet, wodurch sich Zustandsregler für Systeme beliebiger Dimension unter weniger restriktiven Annahmen herleiten lassen.

Anders als der weit verbreitete Einschrittentwurf baut der hier vorgeschlagene sukzessive Mehrschrittentwurf unmittelbar auf dem Integrator-Backstepping auf, indem er explizit die strenge Rückkopplungsform der ODEPDE-ODE-Systeme berücksichtigt. Bekanntlich nutzt das Integrator-Backstepping die genannte Systemstruktur zur schrittweisen Konstruktion von Ljapunov-Funktionen, um auf diese Art Teilsystem für Teilsystem zu stabilisieren und im letzten Schritt die Zustandsrückführung zu gewinnen. Dieses Vorgehen kam auch beim passivitätsbasierten Reglerentwurf für einen Brückenkran mit schweren Ketten in [18] zum Einsatz. Allgemein lässt sich die Grundidee des Integrator-Backsteppings direkt auf die Stabilisierung von ODE-PDE-ODE-Systemen übertragen, wobei durch die Wahl eines kaskadierten Zielsystems, also einer Hintereinanderschaltung der Teilsysteme in den transformierten Koordinaten, auf die für verteilt-parametrische Systeme wesentlich komplexere Ljapunov-Theorie verzichtet werden kann. Letztlich wird die Backstepping-Methode für gekoppelte Systeme dadurch $\mathrm{zu}$ ihren Ursprüngen im Integrator-Backstepping zurückgeführt, was wiederum durch die eindeutige Klärung der schwierigen Frage nach der Wahl einer jeden Transformation eine Systematisierung des Mehrschrittentwurfs in [7] und [6] ermöglicht.
Im Folgenden werden lineare ODE-PDE-ODE-Systeme mit Randaktuierung und örtlich eindimensionalen parabolischen oder hyperbolischen PDEs betrachtet. Auf diese Art schließen die Ergebnisse sowohl PDE-ODE- als auch ODE-PDE-Systeme ein, bei denen die Stellgröße einerseits auf die ODE und andererseits direkt am Rand der PDE wirkt. Dem eigentlichen Backstepping-Entwurf ist in Abschnitt 2 eine ausführliche Motivation auf Basis des $\mathrm{Zu}$ sammenhangs zwischen Integrator-Backstepping und der Backstepping-Methode für PDEs vorangestellt. Die Grundidee wird anschließend in Abschnitt 3 auf parabolische ODE-PDE-ODE-Systeme angewandt, wobei auf Grundlage von deren strenger Rückkopplungsform in jedem der drei Entwurfsschritte sukzessiv Teilsystem für Teilsystem stabilisiert wird, was im letzten Schritt auf eine die Ruhelage des Systems stabilisierende Zustandsrückführung führt. Neben dem Integrator-Backstepping kommt dabei das Konzept des relativen Grads zum Einsatz, wodurch der Entwurf grundsätzlich mit klassischen Methoden der Regelung von endlichdimensionalen Systemen auskommt. Die in den drei Schritten eingesetzten Transformationen bzw. deren Kerne ergeben sich als Lösung von bekannten Kerngleichungen (siehe $[9,10])$ und einfachen Anfangswertproblemen. Da sich parabolische und hyperbolische ODE-PDE-ODE-Systeme strukturell nicht unterscheiden, lässt sich der Entwurf für den hyperbolischen Fall in Abschnitt 4 direkt aus Abschnitt 3 übertragen, wobei auf die Kerngleichungen in [11] und [7] zurückgegriffen werden kann. Letztlich wird in den Abschnitten 5 und 6 aufgezeigt, dass sich der vorgestellte modulare Entwurf direkt auf größere Systemklassen und neue Problemstellungen anwenden lässt, die wesentlich über die Ergebnisse in [7] und [6] hinausgehen.

\section{Motivation und Grundidee}

Die mittlerweile in der Regelungstechnik fest etablierte Backstepping-Methode für PDEs entsprang bekanntlich aus einer Verallgemeinerung des gleichnamigen Verfahrens für ODE-Systeme. So wurde beispielsweise in [4] ein Backstepping-Regler zur Stabilisierung der skalaren Reaktions-Diffusions-Gleichung

$$
\begin{aligned}
\partial_{t} x(z, t) & =\lambda \partial_{z}^{2} x(z, t)+a x(z, t) \\
x(0, t) & =0 \\
x(1, t) & =u(t)
\end{aligned}
$$

mit $\lambda>0$ und $a \in \mathbb{R}$, basierend auf einer Semidiskretisierung von (1) bezüglich des normierten Orts $z \in[0,1]$ durch Bildung des zentralen Differenzenquotienten und damit 
einer endlichdimensionalen Approximation

$$
\begin{aligned}
x_{0}(t) & =0 \\
\dot{x}_{i}(t) & =\lambda \frac{x_{i+1}(t)-2 x_{i}(t)+x_{i-1}(t)}{\Delta z}+a x_{i}(t) \\
x_{n+1}(t) & =u(t),
\end{aligned}
$$

hergeleitet, mit $x_{i}(t)=x(i \Delta z, t), i=1, \ldots, n, \Delta z=\frac{1}{n+1}$.

Das lineare System (2) $n$-ter Ordnung liegt in strenger Rückkopplungsform vor. In einer solchen hängt allgemein die $i$-te Ableitung $\dot{x}_{i}(t), i=1, \ldots, n$, nur von den Komponenten $x_{1}(t), \ldots, x_{i+1}(t)$ mit $x_{n+1}(t)=u(t)$ ab (z.B. [15]), wobei sich in (2) speziell eine Bandstruktur ergibt, die hier jedoch nicht weiter von Interesse ist. Folglich lässt sich durch einen rekursiven Entwurf unter sukzessiver Berücksichtigung von Integratoren, oder kurz Integrator-Backstepping, eine stabilisierende Zustandsrückführung finden (z. B. [16, 1, 15]). Dafür wird im $i$-ten Schritt, $i=1, \ldots, n-1$, die Größe $x_{i+1}(t)$ als virtueller Eingang zur Stabilisierung des Teilsystems mit dem Zustand $\left[x_{1}(t), \ldots, x_{i}(t)\right]^{T}$ durch eine im linearen Fall üblicherweise lineare (virtuelle) Rückführung

$$
\alpha_{i}\left(x_{1}(t), \ldots, x_{i}(t)\right)=\sum_{\ell=1}^{i} c_{i, \ell} x_{\ell}(t)
$$

verwendet und anschließend eine Fehlergröße

$$
\bar{x}_{i+1}(t)=x_{i+1}(t)-\alpha_{i}\left(x_{1}(t), \ldots, x_{i}(t)\right)
$$

eingeführt. Anschaulich gesprochen, werden durch diesen Koordinatenwechsel destabilisierende Terme sukzessive in Richtung der letzten ODE geschoben, wo sie durch geeignete Wahl des Eingangs kompensiert werden können. Aufgrund der Linearität von (2) und der Rückführungen $\alpha_{i}$ ergibt sich dadurch letztlich neben der stabilisierenden Zustandsrückführung $u(t)=\sum_{i=1}^{n} \bar{k}_{i} \bar{x}_{i}(t)=\sum_{i=1}^{n} k_{i} x_{i}(t)$ eine Zustandstransformation der Form

$$
\left[\begin{array}{c}
\bar{x}_{1}(t) \\
\bar{x}_{2}(t) \\
\vdots \\
\bar{x}_{n}(t)
\end{array}\right]=\left[\begin{array}{cccc}
1 & 0 & \cdots & 0 \\
* & \ddots & \ddots & \vdots \\
\vdots & \ddots & \ddots & 0 \\
* & \cdots & * & 1
\end{array}\right]\left[\begin{array}{c}
x_{1}(t) \\
x_{2}(t) \\
\vdots \\
x_{n}(t)
\end{array}\right] .
$$

Wie in [4] gezeigt, lässt sich aus der Zustandsrückführung für die endlichdimensionale Approximation (2) eine Zustandsrückführung für das verteilt-parametrische System (1) zurückgewinnen. Die untere Dreiecksform der Transformationsmatrix motiviert dabei den Ansatz einer Volterra-Integraltransformation

$$
\bar{x}(z, t)=x(z, t)-\int_{0}^{z} k(z, \zeta) x(\zeta, t) \mathrm{d} \zeta,
$$

in der der Transformationskern $k(z, \zeta)$ auf einem (3) entsprechenden, triangulären Gebiet $0<\zeta<z<1$ definiert ist. Die Anwendung von (4) auf die Reaktions-DiffusionsGleichung (1) kann damit auch als verteiltes IntegratorBackstepping mit dem virtuellen Eingang $x(1, t)$ (analog zu $x_{n+1}(t)$ im letzten Backstepping-Schritt für (2)) verstanden werden, das Integral mit dem Kern $k(z, \zeta)$ als (virtuelle) Rückführung. Entsprechend stellt $\bar{x}(z, t)$ eine Fehlergröße dar und der Eingang $u(t)$ muss so gewählt werden, dass $\bar{x}(z, t)$ im durch (4) transformierten System (1) gegen Null geht. Die $n$ Backstepping-Schritte für die Approximation (2) fallen dabei zu einem Schritt für die PDE (1) zusammen.

Diese Grundgedanken lassen sich direkt auf ODE-PDEODE-Systeme übertragen, da sich für diese auf natürliche Weise eine strenge Rückkopplungsform der einzelnen Teilsysteme ergibt. In jedem Backstepping-Schritt wird dabei eines dieser Teilsysteme stabilisiert. Indem die virtuellen Rückführungen so gewählt werden, dass sich im Zielsystem eine Kaskade der einzelnen linearen ODE- und PDETeilsysteme ergibt, folgt die Stabilität des Gesamtsystems aus der Kaskadenstruktur und der Stabilität der Teilsysteme, wobei letztere durch eine einfache Eigenwertvorgabe gesichert wird. Folglich ist keine umfangreiche LjapunovStabilitätsanalyse notwendig.

\section{Der parabolische Fall}

Der parabolische Fall basiert auf dem ODE-PDE-ODESystem in [6], das im Folgenden eingeführt wird. Anschließend werden in drei Entwurfsschritten zuerst die ODE am nicht-aktuierten Rand, dann die PDE auf Grundlage einer klassischen Volterra-Integraltransformation und schließlich die aktuierte ODE durch Überführung des entsprechenden Randsystems auf Byrnes-Isidori-Normalform stabilisiert. Der so erhaltene Regler ist eine Rückführung der Zustände aller Teilsysteme.

\subsection{Problemstellung}

Zentrales Element des parabolischen ODE-PDE-ODESystems sind die $n$ gekoppelten PDEs in

$$
\partial_{t} x(z, t)=\Lambda(z) \partial_{z}^{2} x(z, t)+A(z) x(z, t),
$$

die durch den vom auf das Intervall $[0,1]$ normierten Ort $z$ und der Zeit $t \geq 0$ abhängenden, örtlich verteilten Zustand $x(z, t) \in \mathbb{R}^{n}$ beschrieben werden. ${ }^{1}$ Für $\Lambda(z)=$

1 Die Form (5) hat sich im Rahmen des Backstepping-Entwurfs als vorteilhaft erwiesen (z. B. [9]). Auf allgemeinere DiffusionsAdvektions-Reaktions-Gleichungen geht Abschnitt 5 kurz ein. 
$\operatorname{diag}\left(\lambda_{1}(z), \ldots, \lambda_{n}(z)\right)$ wird eine Diagonalmatrix angenommen, wobei die $C^{2}$-Funktionen $\lambda_{i}, i=1, \ldots, n$, absteigend in der Form $\lambda_{1}(z)>\cdots>\lambda_{n}(z)>0$ geordnet sind. Die PDEs in (5) sind untereinander durch die Matrix $A(z) \in \mathbb{R}^{n \times n}$ gekoppelt, deren Einträge $C^{1}$-Funktionen sind.

Als Randbedingungen für (5) werden hier die RobinBedingungen $^{2}$

$$
\begin{gathered}
\partial_{z} x(0, t)=Q_{0} x(0, t)+C_{0} w_{0}(t) \\
\partial_{z} x(1, t)=Q_{1} x(1, t)+C_{1} w_{1}(t)
\end{gathered}
$$

verwendet, über die die PDEs an den Rändern bei $z=0$ und $z=1$ mit den zwei ODE-Systemen

$$
\begin{aligned}
& \dot{w}_{0}(t)=F_{0} w_{0}(t)+B_{0} x(0, t) \\
& \dot{w}_{1}(t)=F_{1} w_{1}(t)+B_{1} x(1, t)+B u(t)
\end{aligned}
$$

mit den Zuständen $w_{0}(t) \in \mathbb{R}^{n_{0}}$ und $w_{1}(t) \in \mathbb{R}^{n_{1}}$ wechselseitig gekoppelt sind. Derartige Kopplungen treten in der physikalisch-mathematischen Modellbildung ganz natürlich in Folge dynamischer Randbedingungen auf, also wenn eine Randgröße einer Differentialgleichung genügt. Die Dimension des Eingangs $u(t) \in \mathbb{R}^{n}$ wird entsprechend jener des verteilten Zustands angenommen, damit der Rand bei $z=1$ vollständig aktuiert werden kann. Zusätzlich wird für $Q_{0}=\operatorname{diag}\left(q_{0}^{1}, \ldots, q_{0}^{n}\right)$ und $Q_{1}=\operatorname{diag}\left(q_{1}^{1}, \ldots, q_{1}^{n}\right)$ die durchaus physikalisch motivierte Annahme von Diagonalmatrizen getroffen. Die Dimensionen aller nicht näher spezifizierter Matrizen ergeben sich aus dem Kontext.

Für (5)-(9) soll eine Rückführung $u(t)=\mathcal{K}[X(t)]$ des Zustands

$$
X(t)=\left[\begin{array}{l}
w_{0}(t) \\
x(\cdot, t) \\
w_{1}(t)
\end{array}\right] \in \mathcal{X}=\mathbb{R}^{n_{0}} \times L_{2}([0,1])^{n} \times \mathbb{R}^{n_{1}}
$$

entworfen werden, die das System ausgehend von einem beliebigen Anfangszustand $w_{0}(0)=w_{0,0}, x(z, 0)=x_{0}(z)$ und $w_{1}(0)=w_{1,0}$ in der Ruhelage $X(t)=0$ stabilisiert. Dafür werden zwei Annahmen getroffen:

(A1) Das Paar $\left(F_{0}, B_{0}\right)$ ist stabilisierbar.

(A2) Die invarianten Nullstellen des Systems $\left(F_{1}, B, C_{1}\right)$ besitzen negative Realteile.

Mit Hinblick auf die Systemstruktur ist die Notwendigkeit der ersten Annahme sofort einleuchtend, da eine Beeinflussung der $w_{0}$-ODE nur mit Hilfe des Randwerts

2 In Hinblick auf die Ergebnisse in [9] könnten neben RobinRandbedindungen auch Dirichlet- und Neumann-Randbedingungen oder Kombinationen dieser drei Typen betrachtet werden. $x(0, t)$ möglich ist. Die Forderung nach Minimalphasigkeit dient hier lediglich der Vereinfachung der Darstellung. Für nicht-minimalphasige Systeme $\left(F_{1}, B, C_{1}\right)$ wird auf [6] verwiesen. Auch wenn sich darin auf den Fall eines vektoriellen relativen Grads von Eins geschränkt wird, lässt sich das Vorgehen direkt auf beliebige relative Grade verallgemeinern.

Durch Zusammenfassung und Umsortierung der Systemgleichungen (5)-(9) des ODE-PDE-ODE-Systems ergibt sich

$$
\begin{aligned}
\dot{w}_{0}(t) & =F_{0} w_{0}(t)+B_{0} x(0, t) \\
\partial_{z} x(0, t) & =Q_{0} x(0, t)+C_{0} w_{0}(t) \\
\partial_{t} x(z, t) & =\Lambda(z) \partial_{z}^{2} x(z, t)+A(z) x(z, t) \\
\partial_{z} x(1, t) & =Q_{1} x(1, t)+C_{1} w_{1}(t) \\
\dot{w}_{1}(t) & =F_{1} w_{1}(t)+B_{1} x(1, t)+B u(t) .
\end{aligned}
$$

Aus dieser Darstellung und insbesondere der kompakten Notation

$$
\dot{X}(t)=\underbrace{\left[\begin{array}{ccc}
F_{0} & B_{0} \delta_{0} & 0 \\
0 & \Lambda \partial_{z}^{2}+A & 0 \\
0 & B_{1} \delta_{1} & F_{1}
\end{array}\right]}_{\mathcal{A}} X(t)+\underbrace{\left[\begin{array}{l}
0 \\
0 \\
B
\end{array}\right]}_{\mathcal{B}} u(t)
$$

mit dem Zustand aus (10), der punktförmigen Auswertung $\delta_{z} x(\cdot, t)=x(z, t)$ und dem Definitionsbereich

$$
\begin{array}{r}
D(\mathcal{A})=\left\{X \in \mathcal{X}: x \in H_{2}([0,1])^{n}, \partial_{z} x(0)=Q_{0} x(0)\right. \\
\left.+C_{0} w_{0}, \partial_{z} x(1)=Q_{1} x(1)+C_{1} w_{1}\right\}
\end{array}
$$

des Operators $\mathcal{A}$ wird ersichtlich, dass das parabolische ODE-PDE-ODE-System in strenger Rückkopplungsform vorliegt: Auf die $w_{0}$-ODE wirkt als externe Größe nur der PDE-Zustand bei $z=0$, auf die PDE bei $z=1$ hingegen nur der ODE-Zustand $w_{1}(t)$ und schließlich auf die $w_{1}$-ODE der Eingang $u(t)$. Diese Struktur wird im Folgenden genutzt, um sukzessive die einzelnen Teilsysteme zu stabilisieren und durch zusätzliche Kaskadierung die Stabilisierung des Gesamtsystems zu garantieren.

\subsection{Schritt: Stabilisierung der $w_{0}-O D E$}

Im ersten Schritt wird mit

$$
\dot{w}_{0}(t)=F_{0} w_{0}(t)+B_{0} x(0, t)
$$

nur die $w_{0}$-ODE (11a) an jenem Rand $z=0$ betrachtet, der am weitesten vom Eingang $u(t)$ entfernt ist. In dieser, für 
eine bessere Lesbarkeit hier erneut angegeben, ODE übernimmt der Randwert $x(0, t)$ die Rolle eines Eingangs. Basierend auf der Annahme (A1) von Stabilisierbarkeit von $\left(F_{0}, B_{0}\right)$ kann die $w_{0}$-ODE mit dem (virtuellen) Stellgesetz

$$
x(0, t)=\alpha\left(w_{0}(t)\right)=K_{0} w_{0}(t)
$$

stabilisiert werden und es ergibt sich

$$
\dot{w}_{0}(t)=\left(F_{0}+B_{0} K_{0}\right) w_{0}(t)=\tilde{F}_{0} w_{0}(t)
$$

mit einer Hurwitz-Matrix $\tilde{F}_{0}$.

Tatsächlich ist $x(0, t)$ kein Eingang und kann daher nicht frei vorgegeben werden. Wie beim IntegratorBackstepping üblich, wird daher eine Fehlergröße $\tilde{x}(0, t)=$ $x(0, t)-\alpha\left(w_{0}(t)\right)$ als Abweichung zwischen dem Randwert der PDE und dem virtuellen Stellgesetz (13) definiert, welche in Folge durch Vorgabe des (realen) Eingangs $u(t) \mathrm{zu}$ Null gemacht werden soll. Daraus motiviert sich die Transformation

$$
\bar{x}(z, t)=\chi(z, t)-N(z) w_{0}(t)
$$

des PDE-Zustands auf die Fehlergröße $\bar{x}(z, t)$, wobei für die Transformationsmatrix $N(z) \in \mathbb{R}^{n \times n_{0}}$ wegen (13) zumindest

$$
N(0)=K_{0}
$$

gelten soll. Die Anwendung von (14) auf das ODE-PDEODE-System (11) führt auf die transformierte Darstellung

$$
\begin{aligned}
\dot{w}_{0}(t) & =\tilde{F}_{0} w_{0}(t)+B_{0} \bar{x}(0, t) \\
\partial_{z} \bar{x}(0, t)= & Q_{0} \bar{x}(0, t)+\tilde{C}_{0} w_{0}(t) \\
\partial_{t} \bar{x}(z, t)= & \Lambda(z) \partial_{z}^{2} \bar{x}(z, t)+A(z) \bar{x}(z, t) \\
& \quad-N(z) B_{0} \bar{x}(0, t)+\tilde{C}(z) w_{0}(t) \\
\partial_{z} \bar{x}(1, t)= & Q_{1} \bar{x}(1, t)+\tilde{C}_{1} w_{0}(t)+C_{1} w_{1}(t) \\
\dot{w}_{1}(t)= & F_{1} w_{1}(t)+B_{1} \bar{x}(1, t)+B_{1} N(1) w_{0}(t)+B u(t)
\end{aligned}
$$

mit

$$
\begin{aligned}
\tilde{C}_{0} & =C_{0}+Q_{0} K_{0}-N^{\prime}(0) \\
\tilde{C}_{1} & =Q_{1} N(1)-N^{\prime}(1) \\
\tilde{C}(z) & =-N(z) \tilde{F}_{0}+A(z) N(z)+\Lambda(z) N^{\prime \prime}(z) .
\end{aligned}
$$

Nachdem, wie in (16a) erkennbar, das $w_{0}$-Teilsystem exponentiell stabilisiert wurde, ${ }^{3}$ kann durch geeignete Wahl

3 Die Aussage über exponentielle Stabilität bezieht sich auf das autonome System mit $\bar{x}(0, t)=0$. Gleiches gilt im Folgenden bei äquivalenten Betrachtungen.

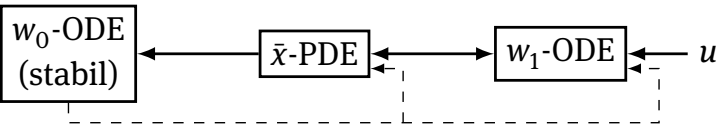

Abb. 1: Struktur des ODE-PDE-ODE-Systems (16) mit der Wahl (17).

von $N(z)$ eine Kaskadierung mit den übrigen Teilsystemen vorbereitet werden. Durch die Wahl

$$
\tilde{C}_{0}=0, \quad \tilde{C}(z)=0
$$

wird erreicht, dass der ODE-Zustand $w_{0}(t)$ nur noch auf das Teilsystem am aktuierten Rand bei $z=1$ rückkoppelt (vgl. strichlierte Pfeile in Abbildung 1). Dieser Einfluss lässt sich später durch geeignete Wahl des Eingangs $u(t)$ kompensieren, weshalb nach dem ersten Transformationsschritt de facto eine Kaskade der exponentiell stabilen $w_{0}$-ODE und des Restsystems vorliegt.

Aus (17) und (15) ergibt sich für die Transformationsmatrix in (14) das Anfangswertproblem

$$
\begin{aligned}
\Lambda(z) N^{\prime \prime}(z)+A(z) N(z)-N(z) \tilde{F}_{0} & =0 \\
N(0) & =K_{0} \\
N^{\prime}(0) & =C_{0}+Q_{0} K_{0},
\end{aligned}
$$

mit (18a) auf $z \in(0,1]$.

Lemma 1. Das Anfangswertproblem (18a) besitzt eine eindeutige stückweise $C^{2}$-Lösung $N(z)$.

Unter Verwendung der Eigenwerte und Eigenvektoren (bzw. Hauptvektoren) von $\tilde{F}_{0}$ lässt sich das matrixwertige Anfangswertproblem für $N(z) \in \mathbb{R}^{n \times n_{0}}$ als $n_{0}$ Anfangswertprobleme im $\mathbb{R}^{n}$ umschreiben, deren Lösung klassisch mit Hilfe der Fundamentalmatrix angegeben werden kann. Weitere Details finden sich in Anhang A.1.

\subsection{Schritt: Stabilisierung der PDE}

Wie in Abschnitt 2 motiviert, lässt sich das IntegratorBackstepping durch eine Volterra-Integraltransformation (4) auf eine PDE übertragen. Dieser zweite Schritt stimmt folglich in den Grundzügen mit dem BacksteppingEntwurf für reine PDE-Systeme, also ohne Ankopplung von ODEs an den Rändern, überein (z. B. [9]). Zur Stabilisierung des PDE-Teilsystems (16b)-(16d) wird entsprechend die Backstepping-Transformation

$$
\tilde{x}(z, t)=\bar{x}(z, t)-\int_{0}^{z} K(z, \zeta) \bar{x}(\zeta, t) \mathrm{d} \zeta=\mathcal{T}[\bar{x}(t)](z)
$$

mit dem $\operatorname{Kern} K(z, \zeta) \in \mathbb{R}^{n \times n}$ und der zugehörigen inversen Abbildung

$$
\bar{x}(z, t)=\tilde{x}(z, t)+\int_{0}^{z} K_{I}(z, \zeta) \tilde{x}(\zeta, t) \mathrm{d} \zeta=\mathcal{T}^{-1}[\tilde{x}(t)](z)
$$


angesetzt. Aus der Identität $\tilde{x}(t)=\mathcal{T}\left[\mathcal{T}^{-1}[\tilde{x}(t)]\right]$, also durch Einsetzen von (20) in (19), ergibt sich die sogenannte Reziprozitätsbeziehung

$$
K_{I}(z, \zeta)=K(z, \zeta)+\int_{\zeta}^{z} K(z, \bar{\zeta}) K_{I}(\bar{\zeta}, \zeta) \mathrm{d} \bar{\zeta}
$$

aus der sich der Kern $K_{I}(z, \zeta) \in \mathbb{R}^{n \times n}$ durch Fixpunktiteration ergibt.

Einsetzen von (19) in das PDE-Teilsystem (16b)-(16d) führt auf

$$
\begin{aligned}
& \partial_{z} \tilde{x}(0, t)=0 \\
& \begin{aligned}
\partial_{t} \tilde{x}(z, t)= & \Lambda(z) \partial_{z}^{2} \tilde{x}(z, t)-\mu_{c} \tilde{x}(z, t)+A_{0}(z) \tilde{x}(0, t) \\
\partial_{z} \tilde{x}(1, t)= & \tilde{Q}_{1} \tilde{x}(1, t)+\tilde{C}_{1} w_{0}(t)+C_{1} w_{1}(t) \\
& \quad+\int_{0}^{1}\left[\tilde{Q}_{1} K(1, z)-\partial_{z} K(1, z)\right] \bar{x}(z, t) \mathrm{d} z
\end{aligned}
\end{aligned}
$$

mit $\tilde{Q}_{1}=Q_{1}-K(1,1)$, sofern der Transformationskern $K(z, \zeta)$ die Kerngleichungen

$$
\begin{aligned}
& \Lambda(z) \partial_{z}^{2} K(z, \zeta)-\partial_{\zeta}^{2}(K(z, \zeta) \Lambda(\zeta)) \\
& \quad=K(z, \zeta)\left(A(\zeta)+\mu_{c} I_{n}\right) \\
& \partial_{\zeta} K(z, 0) \Lambda(0)+K(z, 0)\left(\Lambda^{\prime}(0)-\Lambda(0) Q_{0}\right) \\
& \quad=-A_{0}(z)-\mathcal{T}\left[N B_{0}\right](z) \\
& \Lambda(z)\left(\frac{\mathrm{d}}{\mathrm{d} z} K(z, z)+\partial_{z} K(z, z)\right)+K(z, z) \Lambda^{\prime}(z) \\
& \quad+\partial_{\zeta} K(z, z) \Lambda(z)=-\left(A(z)+\mu_{c} I_{n}\right) \\
& K(z, z) \Lambda(z)-\Lambda(z) K(z, z)=0 \\
& K(0,0)=Q_{0}
\end{aligned}
$$

erfüllt, ${ }^{4}$ wobei die PDE (23a) auf dem Gebiet $0<\zeta<z<1$ definiert ist. Die Existenz einer $C^{2}$-Lösung $K(z, \zeta)$ der Kerngleichungen (23) ist in [9, Thm. 3] gezeigt. ${ }^{5}$ Aus der Lösung $K(z, \zeta)$ ergeben sich die Elemente von

$$
A_{0}(z)=\left[\begin{array}{cccc}
0 & \cdots & \cdots & 0 \\
a_{2,1}(z) & \ddots & & \vdots \\
\vdots & \ddots & \ddots & \vdots \\
a_{n, 1}(z) & \cdots & a_{n, n-1}(z) & 0
\end{array}\right] .
$$

Demnach beschreibt nur ein Teil von (23b) Randbedingungen; der Rest definiert die Elemente $a_{i, j}(z)$ von $A_{0}(z)$.

In Hinblick auf (22b) liegt durch die Struktur von $A_{0}(z)$ eine Kaskade der einzelnen $n$ PDEs vor, wobei die PDE von

4 Allgemein bezeichnet $I_{k}$ die Einheitsmatrix im $\mathbb{R}^{k \times k}$. Darüber hinaus gelten $\partial_{z} K(z, z)=\left.\partial_{z} K(z, \zeta)\right|_{\zeta=z}$ und $\partial_{\zeta} K(z, z)=\left.\partial_{\zeta} K(z, \zeta)\right|_{\zeta=z}$ 5 Im Gegensatz zu (23e) gilt $K(0,0)=0$ in [9]. Da diese zusätzliche Diagonalmatrix lediglich die Initialisierung von (23b) ändert, beeinflusst dieser Unterschied die Aussagen über die Existenz und Eindeutigkeit der Lösung in [9] nicht.

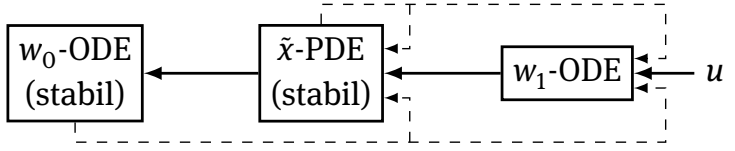

Abb. 2: Struktur des ODE-PDE-ODE-Systems (25).

$\tilde{x}_{i}(z, t), i=2, \ldots, n$ nicht (bzw. nur noch über den aktuierten Rand bei $z=1)$ auf die PDE von $\tilde{x}_{i-1}(z, t)$ rückkoppelt. Setzt man gedanklich den aktuierten Rand mit $\partial_{z} \tilde{x}(1, t)=0$ zu Null, so wird aufgrund der Struktur von (22) klar, dass das kaskadierte PDE-Teilsystem durch geeignete Wahl des Entwurfsparameters $\mu_{c}$ (in der $L_{2}$-Norm) exponentiell stabilisiert wird. Eine solche Wahl ist immer möglich (siehe z. B. [9, Theorem 1]). Die Stabilität des PDE-Teilsystems folgt damit aus der Stabilität der einzelnen $n$ PDEs und ihrer kaskadierten Struktur.

Insgesamt überführt die Integraltransformation (19) mit dem Kern $K(z, \zeta)$ entsprechend (23) das ODE-PDEODE-System (16) auf die Darstellung

$$
\begin{aligned}
\dot{w}_{0}(t)= & \tilde{F}_{0} w_{0}(t)+B_{0} \tilde{x}(0, t) \\
\partial_{z} \tilde{x}(0, t)=0 & \\
\partial_{t} \tilde{x}(z, t)= & \Lambda(z) \partial_{z}^{2} \tilde{x}(z, t)-\mu_{c} \tilde{x}(z, t)+A_{0}(z) \tilde{x}(0, t) \\
\partial_{z} \tilde{x}(1, t)= & \tilde{Q}_{1} \tilde{x}(1, t)+\tilde{C}_{1} w_{0}(t)+C_{1} w_{1}(t) \\
& \quad+\int_{0}^{1} \tilde{K}(z) \tilde{x}(z, t) \mathrm{d} z \\
\dot{w}_{1}(t)=F_{1} w_{1}(t)+B_{1} \tilde{x}(1, t)+B_{1} N(1) w_{0}(t) & \\
& \quad+\int_{0}^{1} \bar{K}(z) \tilde{x}(z, t) \mathrm{d} z+B u(t)
\end{aligned}
$$

mit

$$
\begin{aligned}
\tilde{K}(z) & =\tilde{Q}_{1} K_{I}(1, z)-\partial_{z} K(1, z)-\int_{z}^{1} \partial_{z} K(1, \zeta) K_{I}(\zeta, z) \mathrm{d} \zeta \\
\bar{K}(z) & =B_{1} K_{I}(1, z) .
\end{aligned}
$$

Auf Basis von (22c) wurde dabei der auftretende Integralterm in Abhängigkeit von $\bar{x}(z, t)$ unter Verwendung der Reziprozitätsbeziehung (21) auf die neue Größe $\tilde{x}(z, t)$ überführt. Aus der Struktur von (25) in Abbildung 2 ist zu erkennen, dass das ODE-PDE-ODE-System nach Transformation mit (19), von den strichlierten Rückkopplungseffekten abgesehen, einer Kaskade der zwei bereits stabilisierten Teilsysteme für $w_{0}(t)$ und $\tilde{x}(z, t)$ sowie der im dritten Entwurfsschritt verbleibenden $w_{1}$-ODE entspricht.

\subsection{Schritt: Stabilisierung der $w_{1}-\mathrm{ODE}$}

Der Stabilitätsdiskussion des PDE-Teilsystems im vorangegangenen Abschnitt war die Annahme $\partial_{z} \tilde{x}(1, t)=0$ vorausgegangen. Im letzten Schritt ist daher das Ziel, den Eingang $u(t)$ so zu wählen, dass die (verbleibende) $w_{1}$-ODE 
stabilisiert wird und gleichzeitig $\partial_{z} \tilde{x}(1, t) \rightarrow 0$ für $t \rightarrow \infty$ gilt. Tatsächlich handelt es sich dabei um ein klassisches Problem der Regelung linearer und nichtlinearer Systeme endlicher Dimension. Das wird deutlich, wenn (25e) und (25d) in der Form

$$
\begin{aligned}
\dot{w}_{1}(t) & =F_{1} w_{1}(t)+\mathcal{F} v(t)+B u(t) \\
\chi(t) & =C_{1} w_{1}(t)+\mathcal{G} v(t)
\end{aligned}
$$

angeschrieben werden, wobei $\chi(t)=\partial_{z} \tilde{x}(1, t)$ die Rolle eines Ausgangs in Bezug auf die $w_{1}$-ODE übernimmt und die linearen, unbeschränkten Operatoren

$$
\begin{aligned}
\mathcal{F} v(t) & =B_{1} N(1) w_{0}(t)+B_{1} \tilde{x}(1, t)+\int_{0}^{1} \bar{K}(z) \tilde{x}(z, t) \mathrm{d} z \\
\mathcal{G} v(t) & =\tilde{C}_{1} w_{0}(t)+\tilde{Q}_{1} \tilde{x}(1, t)+\int_{0}^{1} \tilde{K}(z) \tilde{x}(z, t) \mathrm{d} z
\end{aligned}
$$

mit $v(t)^{T}=\left[w_{0}(t)^{T}, \tilde{x}(\cdot, t)^{T}\right]$ alle Rückkopplungseffekte der bereits stabilisierten Teilsysteme zusammenfassen.

Zunächst sei $v(t)=0$, wodurch sich aus (26) die klassische Zustandsdarstellung

$$
\begin{aligned}
\dot{w}_{1}(t) & =F_{1} w_{1}(t)+B u(t) \\
\chi(t) & =C_{1} w_{1}(t)
\end{aligned}
$$

ergibt. Die Frage nach einer den Ausgang $\chi(t)$ für $t \rightarrow$ $\infty \mathrm{zu}$ Null machenden Zustandsrückführung, auch als output-zeroing problem bekannt, führt unmittelbar auf das Konzept des relativen Grads und der Byrnes-IsidoriNormalform (z. B. [13] oder [1]). Dafür bezeichne $\left\{r_{1}, \ldots, r_{n}\right\}$ den (vektoriellen) relativen Grad des linearen Mehrgrößensystems (28), mit $\sum_{i=1}^{n} r_{i} \leq n_{1}=\operatorname{dim} w_{1}(t)$. Dann existiert eine (invertierbare) Zustandstransformation

$$
\left[\begin{array}{l}
\xi(t) \\
\eta(t)
\end{array}\right]=T w_{1}(t), \quad \xi(t)^{T}=\left[\xi_{1}(t)^{T}, \ldots, \xi_{n}(t)^{T}\right]
$$

mit $\xi_{i}(t)=\left[\xi_{i}^{1}(t), \ldots, \xi_{i}^{r_{i}}(t)\right]^{T}$ derart, dass (28) auf die Byrnes-Isidori-Normalform

$$
\begin{aligned}
\dot{\xi}_{i}^{j}(t) & =\xi_{i}^{j+1}(t), \quad j=1, \ldots, r_{i}-1 \\
\dot{\xi}_{i}^{r_{i}}(t) & =m_{i}^{T} \xi(t)+s_{i}^{T} \eta(t)+k_{i}^{T} u(t) \\
\dot{\eta}(t) & =P \xi(t)+Q \eta(t) \\
\chi_{i}(t) & =\xi_{i}^{1}(t)
\end{aligned}
$$

überführt werden kann, in der sich Integratorketten der Länge $r_{i}$ für die Komponenten $\chi_{i}(t), i=1, \ldots, n$, des Ausgangs $\chi(t)=\left[\chi_{1}(t), \ldots, \chi_{n}(t)\right]^{T}$ ergeben. ${ }^{6}$ Die Matrizen $P, Q$

6 Der aufmerksame Leser möge sich daran stören, dass (30d) $r_{i} \neq 0$ und damit $n \leq n_{1}$ impliziert. Offensichtlich ermöglicht eine umfangreichere Notation aber auch die Behandlung des Falls $n>n_{1}$. und Vektoren $m_{i}^{T}, s_{i}^{T}, k_{i}^{T}$ ergeben sich aus $F_{1}, B$ sowie $C_{1}$ und werden hier nicht explizit angegeben. Für (30) lässt sich immer eine die $\xi$-Dynamik asymptotisch stabilisierende Zustandsrückführung angeben, was $\lim _{t \rightarrow \infty} \chi(t)=$ 0 impliziert. Das Gesamtsystem (30) ist nur dann asymptotisch stabil, wenn gleiches für die Nulldynamik $\dot{\eta}(t)=$ $Q \eta(t)$ gilt.

Diese bekannten Ergebnisse lassen sich direkt auf die $w_{1}$-ODE in (26) übertragen. Aufgrund der Operatoren $\mathcal{F}$ und $\mathcal{G}$ in (26) treten dabei sowohl in der (29) ersetzenden Zustandstransformation

$$
\left[\begin{array}{l}
\xi(t) \\
\eta(t)
\end{array}\right]=T w_{1}(t)+\left[\begin{array}{c}
\mathcal{V} v(t) \\
0
\end{array}\right]
$$

als auch in der zugehörigen Byrnes-Isidori-Normalform

$$
\begin{aligned}
\dot{\xi}_{i}^{j}(t) & =\xi_{i}^{j+1}(t), \quad j=1, \ldots, r_{i}-1 \\
\dot{\xi}_{i}^{r_{i}}(t) & =m_{i}^{T} \xi(t)+s_{i}^{T} \eta(t)+\mathcal{M}_{i} v(t)+k_{i}^{T} u(t) \\
\dot{\eta}(t) & =P \xi(t)+Q \eta(t)+\mathcal{H} v(t) \\
\chi_{i}(t) & =\xi_{i}^{1}(t)
\end{aligned}
$$

(vgl. (30)) mit $\mathcal{V} v(t), \mathcal{H} v(t)$ und $\mathcal{M}_{i} v(t), i=1, \ldots, n$, zusätzliche Terme auf, die unter anderem Zeitableitungen der Randwerte $\tilde{x}(0, t)$ und $\tilde{x}(1, t)$ bis zur Ordnung $r_{i}-1$ enthalten. Entsprechend hängt auch eine die $\xi$-Dynamik stabilisierende Zustandsrückführung

$$
u(t)=\overline{\mathcal{K}}[\xi(t), \eta(t), v(t)]
$$

von diesen Zeitableitungen ab, deren Realisierung nicht Gegenstand der vorliegenden Betrachtungen ist (siehe z. B. [6]). Die Annahme (A2) sichert, dass $Q$ in (32c) bzw. im geschlossenen Kreis

$$
\begin{aligned}
\dot{\xi}(t) & =R \xi(t) \\
\dot{\eta}(t) & =P \xi(t)+Q \eta(t)+\mathcal{H} v(t) \\
\chi_{i}(t) & =\xi_{i}^{1}(t)
\end{aligned}
$$

eine Hurwitz-Matrix ist ${ }^{7}$ und damit, dass (33) die $w_{1}$-ODE asymptotisch stabilisiert.

Wie in Abbildung 3 illustriert, führt die Zustandsrückführung (33) auf eine kaskadierte Struktur der einzelnen Teilsysteme im geschlossenen Kreis (25a)-(25c), (34). Basierend auf der Hurwitz-Eigenschaft von $R$ in (34a) klingt

7 Es sei daran erinnert, dass diese Annahme nicht notwendig ist. Für den nicht-minimalphasigen Fall wird in [7] ein komplexerer Ansatz für die Transformation (31) so vorgeschlagen, dass $\mathcal{H} v(t)=0$ in der Byrnes-Isidori-Normalform (32) gilt. Dafür wird ein relativer Grad $\left\{r_{1}, \ldots, r_{n}\right\}=\{1, \ldots, 1\}$ angenommen. 


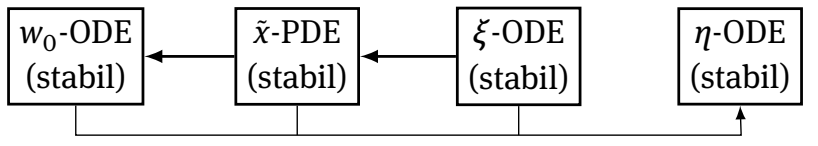

Abb. 3: Struktur des ODE-PDE-ODE-Systems (25a)-(25c), (34).

$\xi(t)$ exponentiell ab, was $\chi(t)=\partial_{z} \tilde{x}(1, t)=0$ für $t \rightarrow \infty$ impliziert und damit in Hinblick auf die Kaskade der $n$ exponentiell stabilen PDEs in (25c) auf $\lim _{t \rightarrow \infty}\|\tilde{x}(t)\|_{L_{2}}=0$ führt. Wegen $\tilde{x}(0, t)=0$ für $t \rightarrow \infty$ und $\tilde{F}_{0}$ Hurwitz geht damit auch $w_{0}(t)$ exponentiell gegen Null. Auf der Basis von $v(t)^{T}=\left[w_{0}(t)^{T}, \tilde{x}(\cdot, t)^{T}\right]=0^{T}$ und $\xi(t)=0$ verbleibt in (34b) nur eine autonome ODE für $\eta(t)$, die in Folge der Hurwitz-Matrix $Q$ exponentiell stabil ist, wodurch zuletzt die exponentielle Stabilität des geschlossenen Kreises (25a)-(25c), (34) gezeigt ist. Aufgrund der Beschränktheit der Transformationen (14), (19) und (31) gilt gleiches für das durch

$$
u(t)=\mathcal{K}[X(t)]
$$

geregelte System (11) bzw. (12) in den Originalkoordinaten $X(t)$, wobei (35) aus (33) unter Verwendung der zuvor genannten drei Transformationen bzw. ihrer Inversen folgt.

\section{Der hyperbolische Fall}

Der für parabolische ODE-PDE-ODE-Systeme aufgezeigte systematische Entwurf von Zustandsrückführungen lässt sich direkt auf den hyperbolischen Fall übertragen, da beide die strukturell gleiche strenge Rückkopplungsform eint. Die Diskussion des Reglerentwurfs für das allgemeine hyperbolische System aus [7] fällt daher kompakter aus.

\subsection{Problemstellung}

Anstelle von (5) werden die $n$ hyperbolischen PDEs

$$
\partial_{t} x(z, t)=\Lambda(z) \partial_{z} x(z, t)+A(z) x(z, t)
$$

betrachtet. Für die Transportgeschwindigkeiten $\lambda_{i} \epsilon$ $C^{1}[0,1], i=1, \ldots, n$, in der Diagonalmatrix $\Lambda(z)=$ $\operatorname{diag}\left(\lambda_{1}(z), \ldots, \lambda_{n}(z)\right)$ gelten

$$
\lambda_{1}(z)>\cdots>\lambda_{p}(z)>0>\lambda_{p+1}(z)>\cdots>\lambda_{n}(z)
$$

und die Diagonalelemente $a_{i i}(z)=0, i=1, \ldots, n$, von $A(z)$ verschwinden. Die Annahme bezüglich der Kopplungsmatrix $A(z)$ lässt sich dabei stets durch eine geeignete $\mathrm{Zu}$ standstransformation erfüllen (siehe [12]) und dient der
Vereinfachung des Backstepping-Entwurfs. Basierend auf der Struktur von $\Lambda(z)$ lassen sich

$$
E_{1}=\left[\begin{array}{c}
I_{p} \\
0
\end{array}\right] \in \mathbb{R}^{n \times p}, \quad E_{2}=\left[\begin{array}{c}
0 \\
I_{m}
\end{array}\right] \in \mathbb{R}^{n \times m},
$$

mit $m=n-p$ definieren, wodurch der Teil $x_{1}(z, t)=$ $E_{1}^{T} x(z, t) \in \mathbb{R}^{p}$ des Zustands $x(z, t) \in \mathbb{R}^{n}$ den Transport in die negative Richtung der Ortskoordinate $z$ bezeichnet und $x_{2}(z, t)=E_{2}^{T} x(z, t) \in \mathbb{R}^{m}$ jenen in die positive Richtung. Die PDE (36) ist über die Randbedingungen

$$
\begin{aligned}
x_{2}(0, t) & =Q_{0} x_{1}(0, t)+C_{0} w_{0}(t) \\
x_{1}(1, t) & =Q_{1} x_{2}(1, t)+C_{1} w_{1}(t)
\end{aligned}
$$

mit den ODEs

$$
\begin{aligned}
& \dot{w}_{0}(t)=F_{0} w_{0}(t)+B_{0} x_{1}(0, t) \\
& \dot{w}_{1}(t)=F_{1} w_{1}(t)+B_{1} x_{2}(1, t)+B u(t)
\end{aligned}
$$

gekoppelt. Im Gegensatz zum parabolischen Fall unterliegen die Matrizen $Q_{0}$ und $Q_{1}$ keinen strukturellen Einschränkungen. Für die Vollaktuierung des Randsystems bei $z=1$ (siehe (38) und (40)) gelte $u(t) \in \mathbb{R}^{p}$ für den Eingang. Darüber hinaus sind weiterhin $w_{0}(t) \in \mathbb{R}^{n_{0}}$ und $w_{1}(t) \in \mathbb{R}^{n_{1}}$ die Zustände der ODEs (39) und (40) mit den Anfangszuständen $w_{0}(0)=w_{0,0}$ und $w_{1}(0)=w_{1,0}$ sowie $x(z, 0)=x_{0}(z)$ für $(36)$.

Fasst man die Gleichungen (36)-(40) zusammen,

$$
\begin{aligned}
\dot{w}_{0}(t) & =F_{0} w_{0}(t)+B_{0} x_{1}(0, t) \\
x_{2}(0, t) & =Q_{0} x_{1}(0, t)+C_{0} w_{0}(t) \\
\partial_{t} x(z, t) & =\Lambda(z) \partial_{z} x(z, t)+A(z) x(z, t) \\
x_{1}(1, t) & =Q_{1} x_{2}(1, t)+C_{1} w_{1}(t) \\
\dot{w}_{1}(t) & =F_{1} w_{1}(t)+B_{1} x_{2}(1, t)+B u(t),
\end{aligned}
$$

so wird ersichtlich, dass auch das hyperbolische ODEPDE-ODE-System in strenger Rückkopplungsform vorliegt. Dies geht insbesondere aus der abstrakten Zustandsdarstellung

$$
\dot{X}(t)=\underbrace{\left[\begin{array}{ccc}
F_{0} & B_{0} E_{1}^{T} \delta_{0} & 0 \\
0 & \Lambda \partial_{z}+A & 0 \\
0 & B_{1} E_{2}^{T} \delta_{1} & F_{1}
\end{array}\right]}_{\mathcal{A}} X(t)+\underbrace{\left[\begin{array}{l}
0 \\
0 \\
B
\end{array}\right]}_{\mathcal{B}} u(t)
$$

hervor, in der der Zustand $X(t)$ wie in (10) definiert ist und

$$
\begin{array}{r}
D(\mathcal{A})=\left\{X \in \mathcal{X}: x \in H_{1}([0,1])^{n}, x_{2}(0)=Q_{0} x_{1}(0)\right. \\
\left.+C_{0} w_{0}, x_{1}(1)=Q_{1} x_{2}(1)+C_{1} w_{1}\right\}
\end{array}
$$

für den Definitionsbereich von $\mathcal{A}$ gilt. Man erkennt leicht, dass die Operatoren $\mathcal{A}$ und $\mathcal{B}$ strukturell mit jenen im parabolischen Fall übereinstimmen (vgl. (12)). Entsprechend 
wird im Folgenden für den Entwurf einer Zustandsrückfühung $u(t)=\mathcal{K}[X(t)]$ analog zu Abschnitt 3 von den Annahmen (A1) und (A2) für Stabilisierbarkeit des Paars $\left(F_{0}, B_{0}\right)$ und Minimalphasigkeit von $\left(F_{1}, B, C_{1}\right)$ ausgegangen. Im nicht-minimalphasigen Fall wird auf [7] verwiesen.

\subsection{Schritt: Stabilisierung der $w_{0}-O D E$}

Im ersten Schritt erlaubt die Wahl des virtuellen Eingangs $x_{1}(0, t)=K_{0} w_{0}(t)$ die Stabilisierung der ODE (41a), wobei die resultierende Systemmatrix $\tilde{F}_{0}=F_{0}+B_{0} K_{0}$ wegen (A1) Hurwitz ist. Wie in (14) definiert man basierend darauf den Fehlerzustand

$$
\bar{x}(z, t)=x(z, t)-N(z) w_{0}(t)
$$

mit der Transformationsmatrix $N(z) \in \mathbb{R}^{n \times n_{0}}$, für die zumindest $E_{1}^{T} N(0)=K_{0}$ gelten muss. Fordert man weiterhin, dass das transformierte System eine Struktur wie in Abbildung 1 aufweisen soll, also dass der ODE-Zustand $w_{0}(t)$ nur noch am aktuierten Rand bei $z=1$ rückkoppeln soll, ergibt sich für $N(z)$ das Anfangswertproblem

$$
\begin{aligned}
\Lambda(z) N^{\prime}(z)+A(z) N(z)-N(z) \tilde{F}_{0} & =0 \\
E_{1}^{T} N(0) & =K_{0} \\
E_{2}^{T} N(0) & =C_{0}+Q_{0} K_{0},
\end{aligned}
$$

mit (45a) auf $z \in(0,1]$.

Lemma 2. Das Anfangswertproblem (45) besitzt eine eindeutige stückweise $C^{1}$-Lösung $N(z)$.

Erneut basiert die Lösungsberechnung auf einem Eigenwertproblem für $\tilde{F}_{0}$ (siehe Anhang A.2).

Mit der Lösung $N(z)$ von (45) transformiert (44) das System (41) auf die Form

$$
\begin{aligned}
\dot{w}_{0}(t) & =\tilde{F}_{0} w_{0}(t)+B_{0} \bar{x}_{1}(0, t) \\
\bar{x}_{2}(0, t) & =Q_{0} \bar{x}_{1}(0, t) \\
\partial_{t} \bar{x}(z, t) & =\Lambda(z) \partial_{z} \bar{x}(z, t)+A(z) \bar{x}(z, t)-N(z) B_{0} \bar{x}_{1}(0, t)
\end{aligned}
$$$$
\bar{x}_{1}(1, t)=Q_{1} \bar{x}_{2}(1, t)+\tilde{C}_{1} w_{0}(t)+C_{1} w_{1}(t)
$$$$
\dot{w}_{1}(t)=F_{1} w_{1}(t)+B_{1} \bar{x}_{2}(1, t)+\bar{B}_{1} w_{0}(t)+B u(t)
$$

mit

$$
\tilde{C}_{1}=\left[Q_{1} E_{2}^{T}-E_{1}^{T}\right] N(1), \quad \bar{B}_{1}=B_{1} E_{2}^{T} N(1) .
$$

Die $w_{0}$-ODE (46a) ist exponentiell stabil und abgesehen von der Rückwirkung bei $z=1$, die später durch geeignete Wahl des Eingangs kompensiert werden kann, mit dem Restsystem (46b)-(46e) kaskadiert (vgl. Abbildung 1).

\subsection{Schritt: Stabilisierung der PDE}

Zur Stabilisierung des PDE-Teilsystems (46b)-(46d) wird wie im parabolischen Fall eine Integraltransformation

$$
\tilde{x}(z, t)=\bar{x}(z, t)-\int_{0}^{z} K(z, \zeta) \bar{x}(\zeta, t) \mathrm{d} \zeta=\mathcal{T}[\bar{x}(t)](z)
$$

angesetzt und ein Kern $K(z, \zeta) \in \mathbb{R}^{n \times n}$ so gesucht, dass sich in der transformierten Darstellung eine Kaskade der einzelnen PDEs ergibt. Genügt $K(z, \zeta)$ den Kerngleichungen

$$
\begin{aligned}
& \Lambda(z) \partial_{z} K(z, \zeta)+\partial_{\zeta}(K(z, \zeta) \Lambda(\zeta))=K(z, \zeta) A(\zeta) \\
& K(z, 0) \Lambda(0)\left[E_{1}+E_{2} Q_{0}\right]=A_{0}(z)+\mathcal{T}\left[N B_{0}\right](z) \\
& K(z, z) \Lambda(z)-\Lambda(z) K(z, z)=A(z),
\end{aligned}
$$

in denen (48a) auf $0<\zeta<z<1$ definiert ist, so überführt (47) das System (46) auf die Darstellung

$$
\begin{aligned}
\dot{w}_{0}(t)= & \tilde{F}_{0} w_{0}(t)+B_{0} \tilde{x}_{1}(0, t) \\
\tilde{x}_{2}(0, t)= & Q_{0} \tilde{x}_{1}(0, t) \\
\partial_{t} \tilde{x}(z, t)= & \Lambda(z) \partial_{z} \tilde{x}(z, t)+A_{0}(z) \tilde{x}_{1}(0, t) \\
\tilde{x}_{1}(1, t)= & Q_{1} \tilde{x}_{2}(1, t)+\tilde{C}_{1} w_{0}(t)+C_{1} w_{1}(t) \\
& \quad+\int_{0}^{1} \tilde{K}(z) \tilde{x}(z, t) \mathrm{d} z \\
\dot{w}_{1}(t)= & F_{1} w_{1}(t)+B_{1} \tilde{x}_{2}(1, t)+\bar{B}_{1} w_{0}(t)+B u(t) \\
& \quad+\int_{0}^{1} \bar{K}(z) \tilde{x}(z, t) \mathrm{d} z,
\end{aligned}
$$

wobei sich

$$
\begin{aligned}
\tilde{K}(z) & =\left[Q_{1} E_{2}^{T}-E_{1}^{T}\right] K_{I}(1, z) \\
\bar{K}(z) & =B_{1} E_{2}^{T} K_{I}(1, z)
\end{aligned}
$$

aus der Reziprozitätsbeziehung (21) unter Verwendung der zu (47) inversen Transformation (vgl. (20)) ergeben. Die Matrix

$$
A_{0}(z)=\left[\begin{array}{l}
A_{1}(z) \\
A_{2}(z)
\end{array}\right]
$$

in (49c) setzt sich aus einer strengen unteren Dreiecksma$\operatorname{trix} A_{1}(z) \in \mathbb{R}^{p \times p}$ (analog zu $A_{0}(z)$ in (24)) und einer Matrix $A_{2}(z) \in \mathbb{R}^{m \times p}$ zusammen. Aufgrund der damit kaskadierten Struktur der PDEs führt ein Randwert $\tilde{x}_{1}(1, t)=0$ nach endlicher Zeit zu $\tilde{x}(z, t)=0$. Damit liegt in (49) eine Systemstruktur wie in Abbildung 2 vor.

Während es sich bei der Transformation (47) um den üblichen Ansatz zur Backstepping-Stabilisierung von gekoppelten hyperbolischen PDEs ohne ODE-Randsysteme handelt (z. B. [11]), stellen (48) eine Verallgemeinerung der bekannten Typen an Kerngleichungen für hyperbolische Systeme dar.

Lemma 3. Die Kerngleichungen (48) besitzen eine eindeutige stückweise $C^{1}$-Lösung $K(z, \zeta)$. 
Tatsächlich kann (48) auf eine Kombination der Kerngleichungen in [11], in denen keine integrale Randbedingung wie in (48b) aufgrund von $\mathcal{T}\left[N B_{0}\right](z)$ auftritt, und jenen in [7, Lemma 6], in denen im Vergleich zu (48) $A(z)=0$ gilt, verstanden werden. Der entsprechende Beweis findet sich in Anhang A.3. Aus der Lösung $K(z, \zeta)$ ergeben sich zum Schluss die von Null verschiedenen Elemente von $A_{0}(z)$ gemäß der entsprechenden Gleichung in (48b), die folglich analog zum parabolischen Fall definitorische Gleichungen darstellen.

\subsection{Schritt: Stabilisierung der $w_{1}-\mathrm{ODE}$}

Im letzten Entwurfsschritt ist das Randsystem bei $z=1$ in (49) so zu stabilisieren, dass $\tilde{x}_{1}(1, t) \rightarrow 0$ für $t \rightarrow \infty$ gilt. Basierend auf den Definitionen

$$
\begin{aligned}
\mathcal{F} v(t)=\bar{B}_{1} w_{0}(t)+B_{1} \tilde{x}_{2}(1, t)+\int_{0}^{1} \bar{K}(z) \tilde{x}(z, t) \mathrm{d} z \\
\mathcal{G} v(t)=\tilde{C}_{1} w_{0}(t)+Q_{1} \tilde{x}_{2}(1, t)+\int_{0}^{1} \tilde{K}(z) \tilde{x}(z, t) \mathrm{d} z
\end{aligned}
$$

lassen sich (49e) und (49d) in der Form (26) mit dem Ausgang $\chi(t)=\tilde{x}_{1}(1, t)$ und $v(t)^{T}=\left[w_{0}(t)^{T}, \tilde{x}(\cdot, t)^{T}\right]$ anschreiben. Mit dem Unterschied, dass $\chi(t) \in \mathbb{R}^{p}$ im hyperbolischen Fall gilt, wohingegen $\chi(t) \in \mathbb{R}^{n}$ in Abschnitt 3.4 ist, ermöglicht die gemeinsame Zustandsdarstellung (26) die direkte Übernahme des verbleibenden Entwurfsalgorithmus aus dem parabolischen Fall. Im Ergebnis ergibt sich eine Zustandsrückführung der Form (33), die dem geschlossenen Kreis im Zielsystem die kaskadierte Struktur in Abbildung (3) aufprägt.

\section{Verallgemeinerungen}

Der für die linearen parabolischen und hyperbolischen ODE-PDE-ODE-Systeme in (11) und (41) dargestellte systematische Backstepping-Entwurf stabilisierender $\mathrm{Zu}$ standsrückführungen lässt sich direkt auf größere Klassen von Systemen übertragen. Einige Erweiterungen liegen auf der Hand.

Da dem dritten Entwurfsschritt in Abschnitt 3.4 und 4.4 die Byrnes-Isidori-Normalform und das Konzept des relativen Grads zugrunde lag, kann die $w_{1}$-ODE auch nichtlinear sein. Beispielsweise lassen sich im parabolischen Fall (11e) und (11d) durch die affine Zustandsdarstellung

$$
\begin{aligned}
\dot{w}_{1}(t) & =f_{1}\left(w_{1}(t), x(1, t)\right)+b\left(w_{1}(t), x(1, t)\right) u(t) \\
\partial_{z} x(1, t) & =c_{1}\left(w_{1}(t), x(1, t)\right)+d\left(w_{1}(t), x(1, t)\right) u(t)
\end{aligned}
$$

mit geeigneten nichtlinearen Funktionen $f_{1}, b, c_{1}$ und $d$, durch die potentiell ein nichtlineares Aktorverhalten beschrieben wird, ersetzen. Aus (52) wird zudem klar, dass der Eingang $u(t)$ auch direkt auf den Randwert der PDE, in (52) $\partial_{z} x(1, t)$, durchgreifen kann.

Allgemein war die strenge Rückkopplungsform der gekoppelten Systeme zentrale Voraussetzung für den sukzessiven Reglerentwurf auf Basis des IntegratorBacksteppings. Entsprechend lassen sich die Systemgleichungen (11) und (41) um solche Ausdrücke erweitern, die die Form der Operatoren $\mathcal{A}$ und $\mathcal{B}$ in (12) und (42) nicht zerstören. Das schließt eine Abhängigkeit der PDE und des Randsystems bei $z=1$ sowohl vom ODE-Zustand $w_{0}(t)$ als auch vom PDE-Zustand $x(z, t)$ selbst in integraler Form ein, wobei der PDEZustand für die Rückwirkung auf die PDE eine Gewichtung über dem Intervall $[0, z]$ und für das Randsystem über $[0,1]$ erfährt. Die PDE geht also in eine partielle Integro-Differentialgleichung über, wodurch sich eine größere Klasse von (chemischen) Prozessen beschreiben lässt. Definiert man mit $\mathcal{J}$ einen Integraloperator $\left(\mathcal{J}_{K} x\right)(z)=\int_{0}^{z} K(z, \zeta) x(\zeta) \mathrm{d} \zeta$ für eine Funktion $K:[0,1] \times[0,1] \rightarrow \mathbb{R}^{n \times n}$ und verwendet weiterhin die punktförmige Auswertung $\delta_{z} f(\cdot)=f(z)$ für Funktionen in $z \in[0,1]$, dann können beispielsweise die Operatoren $\mathcal{A}$ und $\mathcal{B}$ in (12) durch

$$
\mathcal{A}=\left[\begin{array}{ccc}
F_{0} & B_{0} \delta_{0} & 0 \\
G_{1} & \Lambda \partial_{z}^{2}+A+G_{2} \delta_{0}+\mathcal{J}_{G_{0}} & 0 \\
G_{6} & B_{1} \delta_{1}+G_{7} \delta_{0}+\delta_{1} \mathcal{J}_{G_{4}} & F_{1}
\end{array}\right], \quad \mathcal{B}=\left[\begin{array}{l}
0 \\
0 \\
B
\end{array}\right]
$$

mit dem Definitionsbereich

$$
\begin{array}{rl}
D(\mathcal{A})= & \left\{X \in \mathcal{X}: x \in H_{2}([0,1])^{n},\right. \\
\partial_{z} & x(0)=Q_{0} x(0)+C_{0} w_{0}, \partial_{z} x(1)=Q_{1} x(1) \\
& \left.+C_{1} w_{1}+G_{5} w_{0}+G_{8} x(0)+\left(\mathcal{J}_{G_{3}} x\right)(1)\right\}
\end{array}
$$

von $\mathcal{A}$ und Matrizen $G_{0}(z, \zeta), G_{i}(z), i=1, \ldots, 4, G_{j}, j=$ $5, \ldots, 8$, geeigneter Dimension ersetzt werden.

In diesem Zusammenhang sei auch nochmals erwähnt, dass der systematische Backstepping-Entwurf auch für parabolische PDEs mit anderen als den in (6) und (7) betrachteten, wohlgestellten Randbedingungen anwendbar ist (vgl. Fußnote 2), da die Randbedingungen primär im zweiten, auf den Ergebnissen in [9] basierenden, Entwurfsschritt relevant sind. In [9] wird der Backstepping-Entwurf aber nicht nur für parabolische Systeme mit allgemeinen Randbedingungen gelöst, im Gegensatz zur Diffusions-ReaktionsGleichung in (5) werden zusätzliche Advektionsterme betrachtet, wobei das System durch eine Hopf-Cole- 
Transformation wieder auf die Form (5) überführt werden kann.

\section{Zusammenfassung und Ausblick}

Die Rückbesinnung auf die Ursprünge der BacksteppingMethode im Integrator-Backstepping ermöglichte die sukzessive und systematische Stabilisierung gekoppelter hyperbolischer und parabolischer ODE-PDE-ODE-Systeme. Dem endlichdimensionalen Fall entsprechend wurden dabei destabilisierende Terme durch die einzelnen Teilsysteme hin zum aktuierten Rand geschoben, wo sie durch die Stellgröße kompensiert werden konnten. Die Wahl von kaskadierten Zielsystemen machte dabei umfangreiche Stabilitätsbeweise im Rahmen der Ljapunov-Theorie überflüssig. Im Ergebnis stimmen die entworfenen Zustandsrückführungen im Wesentlichen mit jenen in [7] und [6] überein, zumindest insofern, wie von den gleichen Annahmen und Systemen ausgegangen wird. Im Entwurf ergeben sich jedoch Unterschiede in der Anzahl der Transformationsschritte und deren Reihenfolge. In Folge dessen hängen die mit (18) und (45) vergleichbaren Anfangswertprobleme in [7] nicht von der Matrix $A(z)$ ab, was sie im hyperbolischen Fall einer analytischen Lösung zugänglich macht.

Abschnitt 5 hat zudem einige Systemklassen aufgezeigt, auf die sich die vorgestellte Backstepping-Methodik direkt übertragen lässt. Da der sukzessive Entwurf modular ist und insbesondere im zweiten Schritt auf bekannte Kerngleichungen zurückgreift, ist auch eine Kombination mit anderen Ergebnissen naheliegend. So wird beispielsweise in [3] ein modifiziertes Zielsystem für ein hyperbolisches System zweiter Ordnung so vorgeschlagen, dass die Robustheit gegenüber Totzeiten in der PDE gegeben ist. Auch die Untersuchung eines nichtlinearen PDETeilsystems auf der Basis von [19] sollte Gegenstand weiterer Arbeiten sein.

Letztlich erfordert die Realisierung der entworfenen und von der Kenntnis des vollständigen Zustands ausgehenden Regler die Schätzung eben dieser Größen in einem Beobachter, wobei nur von der Messung von (örtlich konzentrierten) Größen des Systems ausgegangen werden kann. Dafür liegen in $[8,7]$ und [6] bereits zahlreiche Ergebnisse vor. Diese lassen sich durch Dualisierung des hier betrachteten Entwurfs von Zustandsrückführungen verallgemeinern und zugleich systematisieren.

Finanzierung: Die Arbeit wurde im Rahmen des österreichischen COMET-K1-Programms (Nr. 854184) durch das Pro ${ }^{2}$ Future-Kompetenzzentrum unterstützt.

\section{Anhang A. Beweise}

\section{A.1 Lösung des Anfangswertproblems (18)}

Es sei $\varphi_{i(k)} \in \mathbb{R}^{n_{0}}$ der Hauptvektor $k$-ter Stufe zum Eigenwert $\mu_{i}$ der Matrix $\tilde{F}_{0}$. Dann gilt

$$
\begin{aligned}
& \tilde{F}_{0} \varphi_{i(1)}=\mu_{i} \varphi_{i(1)} \\
& \tilde{F}_{0} \varphi_{i(k)}=\mu_{i} \varphi_{i(k)}+\varphi_{i(k-1)}, \quad k=2, \ldots, l_{i}
\end{aligned}
$$

für $i=1, \ldots, r$, mit der Summe $\sum_{i=1}^{r} l_{i}=n_{0}$ entsprechend der Dimension von $\tilde{F}_{0}$. Folglich bezeichnet $r$ die Anzahl der Hauptvektorketten und $l_{i}$ deren Länge für einen Eigenwert $\mu_{i}$.

Durch Multiplikation von (18) mit $\varphi_{i(k)}$ von rechts lässt sich das matrixwertige Problem in ein vektorwertiges überführen und mit $n_{i(k)}(z)=N(z) \varphi_{i(k)}$ ergibt sich

$$
\begin{aligned}
\Lambda(z) n_{i(k)}^{\prime \prime}(z) & =-A(z) n_{i(k)}(z)+\mu_{i} n_{i(k)}(z)+n_{i(k-1)}(z) \\
n_{i(k)}(0) & =K_{0} \varphi_{i(k)} \\
n_{i(k)}^{\prime}(0) & =\left(C_{0}+Q_{0} K_{0}\right) \varphi_{i(k)}
\end{aligned}
$$

$k=1, \ldots, l_{i}$, wobei $n_{i(0)}(z)=0$ definiert wird. Daraus folgt unmittelbar die Darstellung

$$
\begin{aligned}
& m_{i(k)}^{\prime}(z)=\bar{A}_{i}(z) m_{i(k)}(z)+D(z) m_{i(k-1)}(z) \\
& m_{i(k)}(0)=\left[\begin{array}{c}
K_{0} \\
C_{0}+Q_{0} K_{0}
\end{array}\right] \varphi_{i(k)}
\end{aligned}
$$

erster Ordnung mit

$$
m_{i(k)}(z)=\left[\begin{array}{l}
n_{i(k)}(z) \\
n_{i(k)}^{\prime}(z)
\end{array}\right] \in \mathbb{R}^{2 n}, \quad D(z)=\left[\begin{array}{cc}
0 & 0 \\
\Lambda^{-1}(z) & 0
\end{array}\right]
$$

und

$$
\bar{A}_{i}(z)=\left[\begin{array}{cc}
0 & I_{n} \\
\Lambda^{-1}(z)\left(\mu_{i} I_{n}-A(z)\right) & 0
\end{array}\right]
$$

Da die Elemente der Matrix $\bar{A}_{i}(z)$ stückweise $C^{1}$-Funktionen sind (vgl. Abschnitt 3.1), garantieren Standardergebnisse für lineare zeitvariante Systeme die Existenz einer stückweisen $C^{1}$-Lösung

$$
m_{i(k)}(z)=\Phi_{i}(z, 0) m_{i(k)}(0)+\int_{0}^{z} \Phi_{i}(z, \zeta) D(\zeta) m_{i(k-1)}(\zeta) \mathrm{d} \zeta
$$

wobei die Fundamentalmatrix $\Phi_{i}(z, \zeta)$ der MatrixDifferentialgleichung

$$
\partial_{z} \Phi_{i}(z, \zeta)=\bar{A}_{i}(z) \Phi_{i}(z, \zeta), \quad \Phi_{i}(z, z)=I_{2 n}
$$

genügt (z. B. [14]). So ergeben sich aus (55) für jedes $i=1, \ldots, r$ mit steigendem $k=1, \ldots, l_{i}$ die 
Lösungen $n_{i(k)}(z)$ (vgl. (54)). Fasst man diese in $\Xi=$ $\left[n_{1(1)}, \ldots, n_{1\left(l_{1}\right)}, n_{2(1)}, \ldots, n_{r\left(l_{r}\right)}\right]$ zusammen und definiert mit $\Psi=\left[\varphi_{1(1)}, \ldots, \varphi_{1\left(l_{1}\right)}, \varphi_{2(1)}, \ldots, \varphi_{r\left(l_{r}\right)}\right]$ die Matrix der Hauptvektoren, so ergibt sich aus $N(z)=\Xi(z) \Psi^{-1}$ die Lösung des Anfangswertproblems (18). Da die Komponenten von $n_{i(k)}^{\prime}(z)$ aus $C^{1}$ stammen, sind die Elemente von $N(z)$ stückweise $C^{2}$-Funktionen.

\section{A.2 Lösung des Anfangswertproblems (45)}

Der Hauptvektor $\varphi_{i(k)}$ der Stufe $k$ zum Eigenwert $\mu_{i}$ der Ma$\operatorname{trix} \tilde{F}_{0}$ sei wie in (53) definiert, $i=1, \ldots, r, k=2, \ldots, l_{i}$ mit $\sum_{i=1}^{r} l_{i}=n_{0}$ (vgl. Abschnitt A.1). Die Multiplikation von (45) mit $\varphi_{i(k)}$ führt auf das klassische Anfangswertproblem

$$
\begin{aligned}
& n_{i(k)}^{\prime}(z)=\bar{A}_{i}(z) n_{i(k)}(z)+\Lambda^{-1}(z) n_{i(k-1)}(z) \\
& n_{i(k)}(0)=\left[\begin{array}{c}
K_{0} \\
C_{0}+Q_{0} K_{0}
\end{array}\right] \varphi_{i(k)}
\end{aligned}
$$

für $n_{i(k)}(z)=N(z) \varphi_{i(k)}$, wobei

$$
\bar{A}_{i}(z)=\Lambda^{-1}(z)\left[\mu_{i} I_{n}-A(z)\right]
$$

und wiederum $n_{i(0)}(z)=0$. Da die Einträge von $\bar{A}_{i}(z)$ stückweise $C^{1}$-Funktionen sind, gilt gleiches für die Lösung

$$
n_{i(k)}(z)=\Phi_{i}(z, 0) n_{i(k)}(0)+\int_{0}^{z} \Phi_{i}(z, \zeta) \Lambda^{-1}(\zeta) n_{i(k-1)}(\zeta) \mathrm{d} \zeta,
$$

in der die Fundamentalmatrix $\Phi_{i}(z, \zeta)(56)$ genügt, und damit auch für $N(z)$.

\section{A.3 Lösung der Kerngleichungen (48)}

Die Lösung $K(z, \zeta)$ von (48) kann auf die Lösung bekannter Kerngleichungen zurückgeführt werden. Dafür wird (47) in zwei Transformationen

$$
\begin{aligned}
& \check{x}(z, t)=\bar{x}(z, t)-\int_{0}^{z} K_{1}(z, \zeta) \bar{x}(\zeta, t) \mathrm{d} \zeta=\mathcal{T}_{1}[\bar{x}(t)](z) \\
& \tilde{x}(z, t)=\check{x}(z, t)-\int_{0}^{z} K_{2}(z, \zeta) \check{x}(\zeta, t) \mathrm{d} \zeta=\mathcal{T}_{2}[\check{x}(t)](z)
\end{aligned}
$$

zerlegt, sodass sich für deren Hintereinanderschaltung

$$
\tilde{x}(z, t)=\mathcal{T}_{2}\left[\mathcal{T}_{1}[\bar{x}(t)]\right](z)=\mathcal{T}[\bar{x}(t)](z)
$$

die Zusammenhänge

$$
\begin{aligned}
K(z, \zeta) & =K_{1}(z, \zeta)+K_{2}(z, \zeta)-\int_{\zeta}^{z} K_{2}(z, \bar{\zeta}) K_{1}(\bar{\zeta}, \zeta) \mathrm{d} \bar{\zeta} \\
K_{I}(z, \zeta) & =K_{1, I}(z, \zeta)+K_{2, I}(z, \zeta)+\int_{\zeta}^{z} K_{1, I}(z, \bar{\zeta}) K_{2, I}(\bar{\zeta}, \zeta) \mathrm{d} \bar{\zeta}
\end{aligned}
$$

ergeben. Die inversen Transformationen von (57) mit den Integralkernen $K_{1, I}(z, \zeta) \in \mathbb{R}^{n \times n}$ und $K_{2, I}(z, \zeta) \in \mathbb{R}^{n \times n}$ sind analog zu (20) definiert.

Wählt man den Kern $K_{1}(z, \zeta) \in \mathbb{R}^{n \times n}$ als Lösung der klassischen Kerngleichungen

$$
\begin{aligned}
\Lambda(z) \partial_{z} K_{1}(z, \zeta)+\partial_{\zeta}\left(K_{1}(z, \zeta) \Lambda(\zeta)\right) & =K_{1}(z, \zeta) A(\zeta) \\
K_{1}(z, 0) \Lambda(0)\left[E_{1}+E_{2} Q_{0}\right] & =H_{0}(z) \\
K_{1}(z, z) \Lambda(z)-\Lambda(z) K_{1}(z, z) & =A(z),
\end{aligned}
$$

wobei (59a) auf $0<\zeta<z<1$ definiert ist, dann wird durch die zugehörige Transformation $\mathcal{T}_{1}$ in (57a) im Wesentlichen die verteilte Kopplung der PDE (46c) über die Matrix $A(z)$ entfernt. Auf die Angabe der entsprechenden Darstellung des ODE-PDE-ODE-Systems wird verzichtet. Für (59) ist in [11] die Existenz einer eindeutigen stückweisen $C^{1}$-Lösung $K_{1}(z, \zeta)$ gezeigt, die neben den Randbedindungen in (59) die Vorgabe künstlicher Randwerte voraussetzt (siehe [11] oder [8]), welche wiederum auch für den $\operatorname{Kern} K(z, \zeta)$ in (48) zu wählen sind. Aus der Lösung $K_{1}(z, \zeta)$ ergeben sich die Elemente der Matrix $H_{0}(z)$, die eine Struktur wie in (51) aufweist.

Basierend auf der nicht angegebenen Systemdarstellung mit dem PDE-Zustand $\check{x}(z, t)$ ergibt sich mit der zweiten Transformation $\mathcal{T}_{2}$ in (57b) genau dann (49) mit dem PDE-Zustand $\tilde{x}(z, t)$, wenn $K_{2}(z, \zeta)$ die Kerngleichungen

$$
\begin{aligned}
& \Lambda(z) \partial_{z} K_{2}(z, \zeta)+\partial_{\zeta}\left(K_{2}(z, \zeta) \Lambda(\zeta)\right)=0 \\
& K_{2}(z, 0) \Lambda(0)\left[E_{1}+E_{2} Q_{0}\right]=A_{0}(z)+\mathcal{T}_{2}\left[G-H_{0}\right](z) \\
& K_{2}(z, z) \Lambda(z)-\Lambda(z) K_{2}(z, z)=0
\end{aligned}
$$

löst, in denen abermals (60a) auf $0<\zeta<z<1$ definiert ist. Zudem gilt $G(z)=\mathcal{T}_{1}\left[N B_{0}\right](z)$. Die Lösung von (60) lässt sich direkt auf jene der Kerngleichungen in [7] mit integraler Randbedingung zurückführen. Damit sichert [7, Lemma 6] die Existenz einer eindeutigen stückweisen $C^{1}$-Lösung $K_{2}(z, \zeta)$, aus der sich zudem die Elemente der Matrix $A_{0}(z)$ ergeben. In Folge der Komposition (58) ist damit gezeigt, dass die Lösung $K(z, \zeta)$ von (48) stückweise stetig differenzierbar und eindeutig ist.

\section{Literatur}

1. AdAMY, J.: Nichtlineare Regelungen. Springer-Verlag, 2009.

2. ANFINSEN, H. and O. AAMO: Stabilization of a linear hyperbolic PDE with actuator and sensor dynamics. Automatica, 95:104-111, 2018.

3. Auriol, J., U.J.F. Aarsnes, P. MARTIn and F. Di Meglio: Delay-robust control design for two heterodirectional linear coupled hyperbolic PDEs. IEEE Trans. Autom. Contr., 63:3551-3557, 2018. 
4. BALOgh, A. and M. KRStiC: Infinite dimensional backstepping-style feedback transformations for a heat equation with an arbitrary level of instability. Eur. J. Contr., 8:165-175, 2002.

5. Bou Saba, D., F. Bribiesca-Argomedo, M. Di Loreto and D. EBERHARD: Backstepping stabilization of $2 \times 2$ linear hyperbolic PDEs coupled with potentially unstable actuator and load dynamics. In: Proc. 56th IEEE Conference on Decision and Control (CDC), pages 2498-2503, 2017.

6. DeUtSCHER, J. and N. Gehring: Output feedback control of coupled linear parabolic ODE-PDE-ODE systems. IEEE Trans. Autom. Contr., eingereicht, 2020.

7. DeUtSChER, J., N. GeHring and R. KeRn: Output feedback control of general linear heterodirectional hyperbolic ODE-PDE-ODE systems. Automatica, 95:472-480, 2018.

8. DeUtSChER, J., N. GeHRING and R. KeRn: Output feedback control of general linear heterodirectional hyperbolic PDE-ODE systems with spatially-varying coefficients. Int. J. Contr., 92:2274-2290, 2019.

9. DEUTSCHER, J. and S. KeRSCHBAUM: Backstepping control of coupled linear parabolic PIDEs with spatially varying coefficients. IEEE Trans. Autom. Contr., 63:4218-4233, 2018.

10. DeUTSCHER, J. and S. KeRSCHBAUM: Backstepping für gekoppelte parabolische Systeme mit ortsabhängigen Koeffizienten. at - Automatisierungstechnik, 66:558-572, 2018.

11. Hu, L., R. VAzquez, F. Di Meglio and M. Krstic: Boundary exponential stabilization of 1-D inhomogeneous quasilinear hyperbolic systems. arXiv preprint arXiv:1512.03539, 2015.

12. Hu, L., R. VAzQuez, F. Di MEglio and M. KRStic: Control of homodirectional and general heterodirectional linear coupled hyperbolic PDEs. IEEE Trans. Autom. Control, 61:3301-3314, 2016.

13. ISIDORI, A.: Nonlinear Control Systems. Springer-Verlag, 1995.

14. Kailath, T.: Linear Systems. Prentice Hall, 1980.

15. Krstic, M., I. Kanellakopoulos and P. Кокотovic: Nonlinear and Adaptive Control Design. Wiley, 1995.
16. RUDOLPH, J.: Rekursiver Entwurf stabiler Regelkreise durch sukzessive Berücksichtigung von Integratoren und quasi-statische Rückführungen. at - Automatisierungstechnik, 53:389-399, 2005.

17. TANG, S. and C. XIE: State and output feedback boundary control for a coupled PDE-ODE system. Syst. Contr. Lett., 60:540-545, 2011.

18. THuLL, D., D. WILD and A. KUGI: Infinit-dimensionale Regelung eines Brückenkrans mit schweren Ketten. at Automatisierungstechnik, 53:400-410, 2005.

19. Vazquez, R. and M. KRStic: Control of 1-D parabolic PDEs with Volterra nonlinearities, part 1: design. Automatica, 44:2778-2790, 2008.

20. WANG, J. and M. KRSTIC: Output feedback boundary control of a heat PDE sandwiched between two ODEs. IEEE Trans. Autom. Contr., 64:4653-4660, 2019.

\section{Autoreninformationen}

\section{Dr.-Ing. Nicole Gehring}

Johannes Kepler Universität, Institut für Regelungstechnik und Prozessautomatisierung, Altenberger Straße 69, 4040 Linz, Österreich nicole.gehring@jku.at

Nicole Gehring promovierte 2015 am Lehrstuhl von Prof. J. Rudolph an der Universität des Saarlandes und war anschließend zwei Jahre am Lehrstuhl von Prof. B. Lohmann an der Technischen Universität München tätig. Seit 2017 arbeitet sie als Universitätsassistentin am Institut von Prof. K. Schlacher an der Johannes Kepler Universität Linz. Hauptarbeitsgebiete: lineare verteilt-parametrische Systeme, Backstepping-Entwurf, flachheitsbasierte Methoden. 